\title{
Quality control in routine haemoglobinometry
}

\author{
I. CAVILL
}

From the Department of Haematology, Welsh National School of Medicine, Cardiff

SYNOPSIS An analysis of haemoglobin estimations in a routine laboratory shows that quality control methods based on mean values are unsatisfactory. The use of a cusum method together with control samples is a sensitive and convenient technique for the early discovery of minor deviations in results and it is not dependent on a stable population of patients.

Current methods of intralaboratory quality control in haematology are generally based on an analysis of the routine haematology results. These methods assume a stable patient population. Our results show that this assumption is not valid for haemoglobin estimation. An alternative method, based on control specimens and the cumulative sum (cusum) technique (Woodward and Goldsmith, 1964), is described.

\section{An Analysis of Haemoglobin Results}

A preliminary analysis was made of the haemoglobin results produced in the routine laboratory. The pattern of results was followed over a two-year period in order to evaluate a system of quality control based on the daily routine results. The accuracy of the haemoglobin results produced by the laboratory was assessed in interlaboratory quality control trials organized by $\operatorname{Dr} \mathrm{S}$. M. Lewis.

All the routine results reported from the haematology laboratory during a typical week in January 1969 were analysed. A frequency distribution histogram of 852 results showed a marked skew towards the lower values (Fig. 1). This was to be expected in a sample population which was already selected for some suspected pathological abnormality. The mean and standard deviation of such a distribution are statistically misleading and the results must be transformed to give a normal distribution. The frequency distribution of the square of each haemoglobin value (Fig. 2) was shown to be normal using probability paper (Croxton, 1959). The square root of the mean squared haemoglobin concentration is referred to as the 'normalized' mean.

A normalized mean of the haemoglobin results reported on each day over an eight-week period was Received for publication 17 February 1971. calculated from the squared distribution. During each week the normalized mean was highest on the Monday and showed a steady decline on successive days (Fig. 3). An analysis of variance showed that the variation between the means on successive days was greater than the variation between the means on the same day of each succeeding week $(F=11 \cdot 21$; $P<0.001$ ). The normalized mean for each particular day of the week showed a number of changes during the subsequent five months. On Mondays and Tuesdays the means dropped by an average of $0.1 \mathrm{~g} / 100 \mathrm{ml}$ after two months; on Wednesdays and Fridays there was a mean increase of $0.4 \mathrm{~g} / 100 \mathrm{ml}$; Thursday's mean remained stable while Saturday's showed three separate changes, increasing and decreasing by $0.4 \mathrm{~g} / 100 \mathrm{ml}$ alternately.

Although these changes were unrelated to one another, the pattern of a decreasing daily mean throughout the week was maintained. This pattern could not be related to any weekly cycle of events in the laboratory and appeared to be a function of the changing sample of specimens sent to the laboratory. This, in turn, reflected the weekly round of clinics, admissions, and surgical timetables. These results indicate that the normalized mean of the daily sample of routine results cannot be used as the basis of a quality control system. Alternative methods of quality control which depend on the inclusion of control specimens in the routine procedure were therefore examined.

\section{Control Samples and the Cusum Technique}

The first consideration of any quality control system based on the use of control samples is the stability of the reference preparation. The haemoglobin concentration of whole blood in acid-citratedextrose (ACD) solution stored at $0-4^{\circ} \mathrm{C}$ was measured 


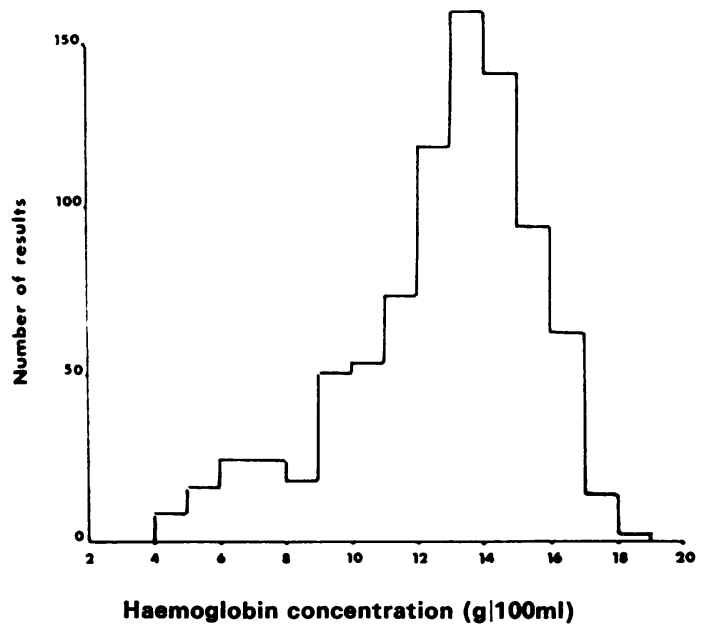

Fig. 1 The frequency distribution of 852 routine haemoglobin results reported by the laboratory during one week.

over a period of one month and found to be stable. Two control samples in ACD were prepared from blood taken from patients having venesection therapy. An artifically high or low haemoglobin concentration was produced either by concentrating the red cells or diluting them with saline. Aliquots, $20 \mathrm{ml}$ of each sample, were dispensed into screwtopped glass containers and stored at $0-4^{\circ} \mathrm{C}$. The haemoglobin concentration of an aliquot of each

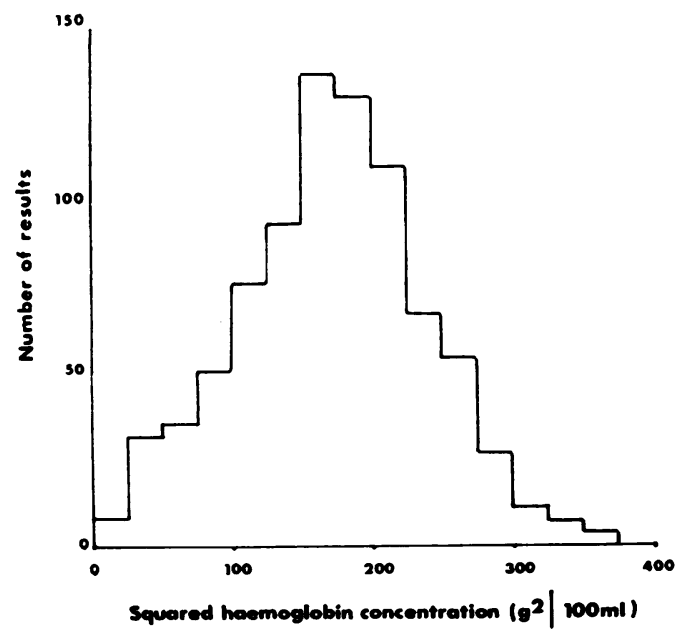

Fig. 2 The frequency distribution of the squared values of 852 routine haemoglobin results reported by the laboratory during one week. sample was measured 10 times and the mean and standard deviation were calculated. An aliquot of each sample was taken from storage each morningo and kept on a Mattburn mixer throughout the day.믐 The haemoglobin concentration of the controp aliquot was estimated after every 20 routine tests $\mathbb{\complement}$ The difference between each estimation and the meany (reference) value was calculated and recorded. The cumulative sum (cusum) of these differences, taking notice of their signs, was plotted against the number of control tests that had been performed. Eacho control sample was used for one week after which as fresh batch was prepared.

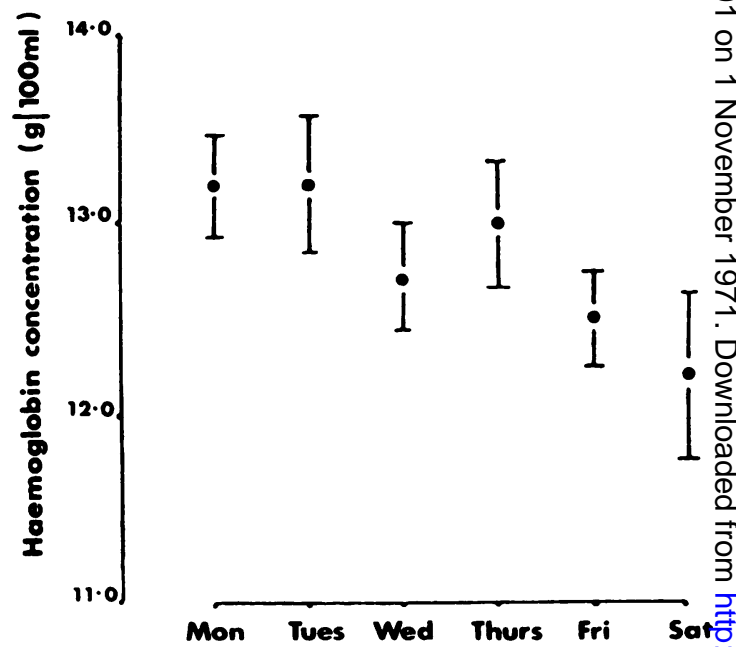

Fig. 3 The mean ( $\pm 1 S D$ ), over an eight-week period, of the normalized mean haemoglobin concentration for each day of the week.

The cusum system was originally developed for the control of industrial chemical processes (Wood $\frac{7}{0}$ ward and Goldsmith, 1964). It is a means of magnifying consistent differences between a referenceN value and subsequent estimates; random changes tend to cancel each other out and in a stable situation? the cusum plot would oscillate about a value of zero. $\omega$ A slight, but consistent, difference between the meane value calculated initially and the results of subs? sequent estimations would produce a cusum of increasing magnitude. If the cusum is plotted on arithmetic graph paper with the interval between each test of the same size as 2 standard deviations on the ordinate then a change of $45^{\circ}$ in the plot is? an indication that a significant deviation has occurred. A single difference between the mean and? 
Haemoglobin

$\mathrm{gm} / 100 \mathrm{ml}$
Fig. 4 The daily normalized mean of the haemoglobin results and the cusum plot of one control sample during one week (Wednesday to Wednesday).
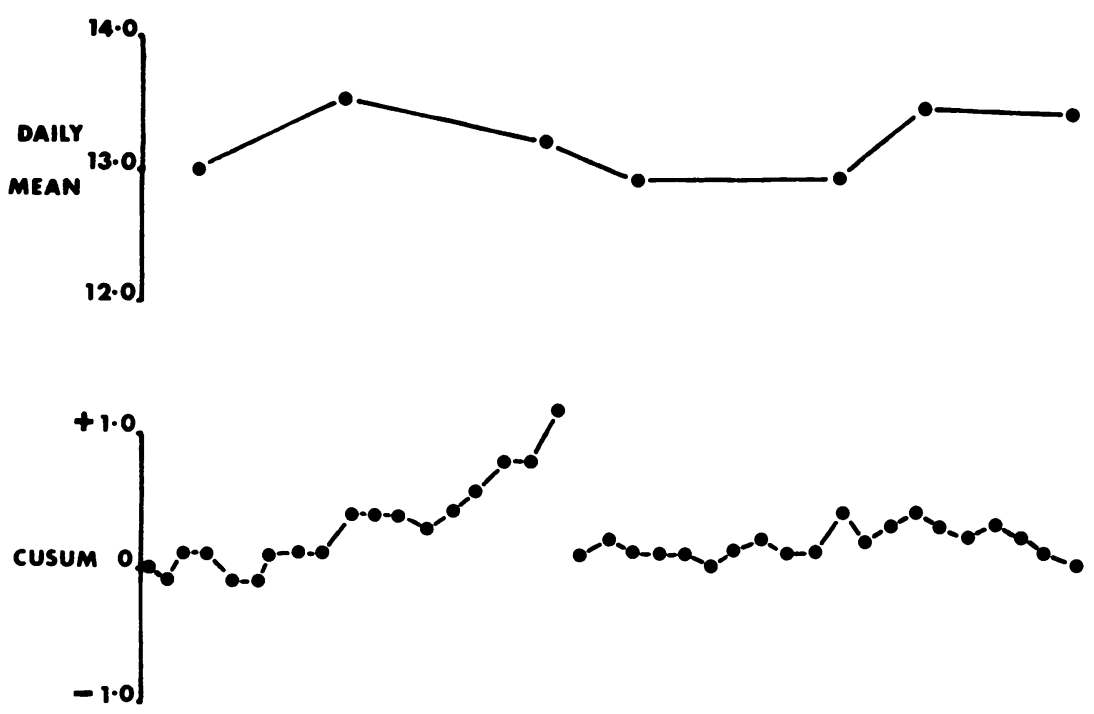

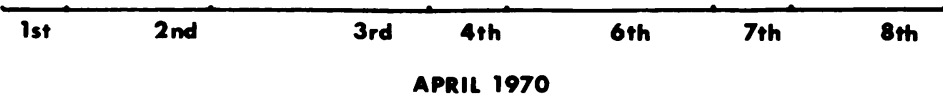

the control estimate which was as great as or greater than three times the standard deviation would also indicate a significant deviation from control. If either of these conditions occurred all routine tests were stopped, the apparatus and solutions were checked, and recalibration was carried out if necessary.

A comparison between daily normalized mean values and the cusum technique was made and the latter was found to be the more sensitive to minor changes. The cusum plot from one control sample during one week is shown in Figure 4. The daily normalized mean of all the routine results showed the typical pattern, decreasing gradually from Monday to Saturday. During the first two days the cusum plot remained stable but on the third day an upward trend developed. At the end of that day it was obvious that the plot was not going to return to the horizontal. Examination of the diluent solutions revealed an accumulation of particles in one of the containers. A clean solution was substituted and the cusum returned to its stable condition. There had been a mean increase of $0.2 \mathrm{~g}$ per $100 \mathrm{ml}$ in each estimate of the control haemoglobin concentration. The daily normalized mean gave no indication of this change.

\section{Discussion}

The most widely used methods of quality control are, at present, based on the daily calculation of the mean of all or part of the day's routine results. This has the disadvantage of having an effect only after all the results have been reported and are past correction. The analysis of the routine results showed that the number plus system (Hoffmann and Waid, 1963) would also have been unsatisfactory as a means of quality control and a similar conclusion has been reached in relation to blood sugar, blood urea, and serum sodium levels (Frankel and Ahlvin, 1967).

The inclusion of control samples in the routine procedure is the only adequate method of quality control for haemoglobinometry. Our choice of two controls every 20 routine samples is arbitrary but appears justified in practice. This $10 \%$ increase in the workload has proved acceptable using a Coulter $\mathrm{S}$ counting system. 
The aim of any system of quality control is to ensure that a result obtained from a patient at a particular time can be meaningfully compared with a result from the same or any other patient at any other time. It is therefore just as important to have an objective measure of the consistency of the results as it is to have a warning of any major deviation. The cusum method of analysis of the control data enables very small changes in the procedure to be detected and corrected before they become of clinical significance. If the cusum plot is steady then this is a positive index that the procedure is under control.

\section{Reference:}

Croxton, F. E. (1959). Elementary Statistics with Applications i Medicine and the Biological Sciences. Dover, New York.

Frankel, S., and Ahlvin, R. C. (1967). An evaluation of the number plus method of quality control. Amer. J. clin. Path., 48, 248-250

Hoffmann, R. G., and Waid, M. E. (1963). The number plus method of quality control of laboratory accuracy. Amer. J. clin. Path.,

40, 263-269.
Woodward, R. H., and Goldsmith, P. L. (1964). Cumulative Sum Techniques. Oliver and Boyd, Edinburgh. 